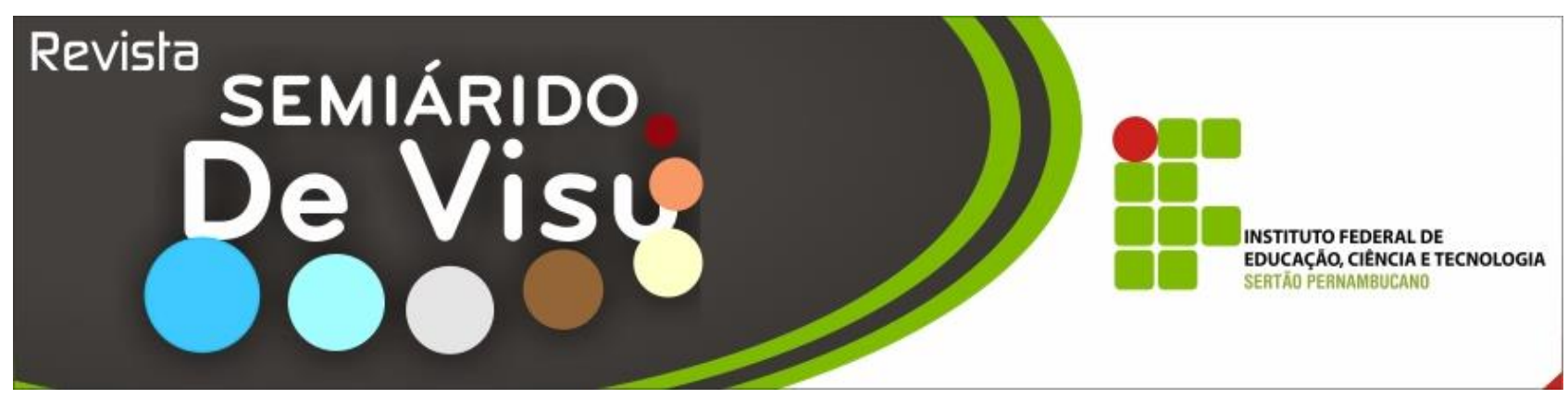

\title{
Avaliação da uniformidade de aplicação de água em sistemas de irrigação do perímetro irrigado Nilo Coelho em Petrolina-PE
}

\author{
Wagner Willen Cavalcanti Araquam¹, Luís Fernando de Souza Magno Campeche²
}

\author{
1.Mestrando em Engenharia Agrícola - Universidade Federal do Vale do São Francisco - UNIVASF, CEP \\ 48902-300, Juazeiro, BA . Fone: (74) 2102-7621 , e-mail: wagnerwillen@ hotmail.com \\ 2 Prof. Doutor, Instituto Federal de Educação, Ciência e Tecnologia do Sertão Pernambucano - IF \\ SERTÃO-PE - zona rural, Petrolina-PE.
}

\begin{abstract}
RESUMO: A horticultura do Vale do São Francisco, principalmente a produção de frutas, submetida a irrigação, é a maior fonte de economia da região e mantém-se em posição de destaque no cenário nacional. Porém, variações nas produtividades têm ocorrido por causa de problemas nos sistemas de irrigação. O objetivo desse trabalho foi avaliar a uniformidade de aplicação de água dos sistemas de irrigação pressurizados presentes no Perímetro Irrigado Nilo Coelho, em Petrolina-PE. Foram selecionados alguns lotes, aleatoriamente, e verificada a vazão em alguns dos emissores. Na análise de dados calculou-se o Coeficiente de Uniformidade Christiansen - CUC, o Coeficiente de Uniformidade de Distribuição - CUD e o Coeficiente Estatístico de Uniformidade - CUE, no qual foi feita a classificação do grau de aceitabilidade. Os sistemas de irrigação localizada (microaspersão e gotejamento) apresentaram os melhores resultados, qualificados assim como eficientes na distribuição de água. Os sistemas de aspersão convencional, devido aos baixos valores obtidos para os coeficientes analisados, foram classificados como inaceitáveis, necessitando de manutenção e/ou redimensionamento.
\end{abstract}

Palavras-chave: Coeficientes de uniformidade, avaliação da irrigação, eficiência.

\section{Evaluation of the uniformity of water application in irrigation systems of irrigated perimeter Nilo Coelho in Petrolina-pe}

\begin{abstract}
The Horticulture of the São Francisco, mainly fruit production, subject to irrigation, is the major source of economy of the region and remains in a prominent position on the national scene. However, variations in productivity have occurred because of problems in irrigation systems. The aim of this study was to evaluate the uniformity of water application of pressurized irrigation systems present in the perimeter Irrigated Nilo Coelho, in Petrolina-PE. Some batches were selected randomly, and verified the flow in some of the emitters. In data analysis we calculated the Christiansen Uniformity Coefficient - CUC, the Coefficient of Uniformity Distribution - CUD and the statistical uniformity coefficient - CUE, in which gone made the classification the degree of acceptability. The located irrigation systems (micro sprinkler and drip) showed the best results, qualified as water efficient distribution. The conventional sprinkler systems due to low values for the coefficients analyzed were classified unacceptable as , requiring maintenance and / or resizing.
\end{abstract}

Keywords: Coefficient of uniformity, evaluation of irrigation, efficiency. 


\section{Introdução}

gricultura irrigada vem
provocando bastante discussão
no meio científico, pela enorme quantidade de água utilizada na produção de alimentos. Christofidis (2006), revela que no mundo, uma área de cerca de 1,532 bilhão de hectares está em produção agrícola, dos quais cerca de 278 milhões de solos sob o domínio de infra-estrutura hídrica de irrigação. A área de $18 \%$ sob cultivo irrigado produz cerca de $44 \%$ da produção total agrícola, enquanto a agricultura de sequeiro responde pelo restante.

De acordo com Gomes (1994), a irrigação é uma prática agrícola de fornecimento de água às culturas, onde e quando os índices pluviométricos, ou qualquer outra forma natural de abastecimento, não são suficientes para suprir as necessidades hídricas das plantas. Constitui-se em uma atividade atualmente de grande importância para manutenção do aspecto econômico comercial da produtividade agrícola em quase todas as regiões e climas do nosso planeta.

A irrigação localizada desponta como uma das contribuições mais promissoras para o desenvolvimento da fruticultura irrigada no Brasil, e mais especificamente no Nordeste (CARVALHO et al., 2006). Dessa forma, é indiscutível a necessidade da irrigação na região do vale do submédio do São Francisco, levando-se em conta a disponibilidade de água do rio, as características edafoclimáticas diversificadas com ocorrência de chuvas irregular e má distribuídas. Promover a racionalização da água, otimizando a produtividade dos cultivos, depende da eficiência do seu uso, visando não só a questão ecológica, mas também econômica. A sustentabilidade deverá superar a escassez e promover uma nova ética aos recursos hídricos, tendo como base a otimização da utilização, controle dos desperdícios, além de promover o desenvolvimento, (TUNDISI, 2005). Portanto, o conhecimento do desempenho e performance de qualquer sistema de irrigação será de suma importância, uma vez que o objetivo principal de um projeto de irrigação é de aumentar a produtividade com o menor custo de produção.

A irrigação tem permitido uma agricultura estável e próspera no Brasil, perante fatores adversos de solo e clima. No entanto, o consumo ocasionado pelas atividades agrícolas no Brasil é responsável por $61 \%$ do consumo de água, (CHRISTOFIDIS, 2006). Assim, relatam alguns pesquisadores (PERI et al.,1979; WALKER, 1979) que a aplicação de água por qualquer sistema de irrigação é naturalmente desuniforme. Mediante a instabilidade dos sistemas de irrigação passa a ser um desafio, manter a estabilidade agrícola disponibilizando uma quantidade mínima necessária para planta, de água e de insumos, e obter uma máxima produção.

Conforme Coelho et al. (2005), o uso eficiente da água de irrigação pode ser alcançado melhorando: a) a estrutura de irrigação então existente, em termos de tipos de cultivo, sistemas de irrigação e gestão do uso de água; b) os métodos de manejo da irrigação e c) as técnicas que permitem aumento da eficiência do uso da água.

Para Santos et al. (2003), a uniformidade de distribuição de água em áreas irrigadas influencia diretamente o manejo, a qualidade, a eficiência e o custo da irrigação, assim como o desempenho da cultura no campo. Para realizar um crescimento uniforme da cultura é requerida que água seja uniformemente aplicada em toda a área.

Nenhum sistema de irrigação é capaz de aplicar água com perfeita uniformidade. A eficiência de aplicação de água no campo pode ser $100 \%$ se o solo encontrar-se vazio e toda a água aplicada tornar-se disponível para as plantas. Entretanto, se a água for aplicada desuniformemente, em algumas áreas a cultura receberá pouca água e em outras podem ocorrer excessos. Os excessos de água aplicados no solo, não disponíveis para a cultura, poderão contribuir para o escoamento superficial ou percolação profunda abaixo da zona radicular. A velocidade do vento, o escoamento e a evaporação também colaboram na redução da eficiência de aplicação. 
Minimizar os efeitos negativos em campo tem sido o grande desafio da irrigação. Embora, em geral, Heermann et al. (1992) afirmam que o aumento da uniformidade de distribuição da água requeira investimentos na melhoria do sistema, em manutenção e em mão-de-obra, para o manejo racional da irrigação.

Tarjuelo et al. (1996) salientam que os benefícios econômicos da irrigação aumentam em função do aumento da uniformidade de distribuição, independentemente do custo da água. Mantovani et al. (1995) mostraram que a relação ótima entre lâmina de irrigação e lâmina de água necessária à cultura do milho depende da uniformidade de distribuição de água e da relação entre o preço do produto e o custo da água.

Conforme Boman (1989), a uniformidade é um indicador da igualdade das taxas de aplicação dentro do diâmetro padrão de um emissor. Uniformidade alta é importante para irrigação em terras arenosas, onde a redistribuição lateral da água é limitada. Excessos de aplicação de água nesses solos resultam freqüientemente em lâmina percolada e lixiviação de nutrientes para fora da zona radicular. A uniformidade elevada pode ser especialmente desejável para fertirrigação ou quimirrigação, visto que as aplicações de nutrientes ou compostos químicos não serão mais uniformes que a distribuição de água.

Conseguinte, é de extrema relevância avaliar um sistema de irrigação após sua fase de instalação e indispensável na manutenção do sistema, verificar a uniformidade de distribuição da água nos emissores. Keller \& Bliesner (1990) comentam que é recomendável, após a instalação de um sistema de irrigação, proceder a testes de campo, com o objetivo de se verificar a adequação da irrigação, recomendando quando necessário, ajustes na operação e, principalmente, no manejo. Esses procedimentos visam maximizar a eficiência do sistema.

Conforme Cabello et al. (1986), os fatores de fabricação medidos pelo coeficiente de variação, os fatores hidráulicos da instalação devidos a pressões diferentes a que estão submetidos os emissores, as obstruções e os efeitos da temperatura da água influenciam na uniformidade de aplicação de água em um sistema de irrigação localizada. E ainda para Matos et al. (1996), vários são os fatores que influenciam na uniformidade de distribuição de água do microaspersor, podendo-se citar: posição do suporte, ângulo de irrigação, tronco e folhagem da árvore, elemento distribuidor, pressão de trabalho, reguladores e amortecedores e vazão nominal.

A avaliação da uniformidade de aplicação da água nos sistemas de irrigação dar-se por vários modelos estatísticos, baseados na variação da vazão, a qual é proporcional à distribuição de pressão ao longo do comprimento do conduto.

A uniformidade de aplicação da água sobre a área irrigada é um dos principais parâmetros para avaliar o desempenho de um sistema de irrigação por aspersão. Pode ser estimada pelo coeficiente de uniformidade. $\mathrm{O}$ conceito de uniformidade de distribuição, UD, foi originalmente apresentado por Keller \& Karmeli (1974), sendo a sua definição baseada entre as vazões mínima e média dos emissores, que é utilizada também para irrigação localizada.

A uniformidade está associada à variabilidade da lâmina de irrigação ao longo da área molhada (FRIZZONE, 1992). Para Bernardo (1995), a uniformidade pode ser expressa por índices ou coeficientes, sendo o mais utilizado o Coeficiente de Uniformidade de Christiansen - CUC (1942). São também utilizados em menor escala o Coeficiente de Uniformidade de Distribuição - CUD proposto por Criddle et al. (1956) e o Coeficiente Estatístico de Uniformidade - CUE, apresentado primeiramente por Wilcox \& Swailes (1947). Sendo assim, a uniformidade de aplicação é a estimativa da porcentagem do total de água aplicada na irrigação por aspersão que atinge a superfície do solo e/ou as plantas.

As principais variáveis que afetam estas perdas são: velocidade do vento, temperatura e umidade relativa do ar. Essa eficiência é de grande importância para o Nordeste e para as 
regiões cuja umidade relativa do ar é muito baixa.

Para Martins (2009) a baixa uniformidade de aplicação de água de sistemas de irrigação localizada se deve em grande parte ao mau dimensionamento do projeto e a ausência de manejo do sistema. Geralmente, reduzidos valores de uniformidade determinam maior consumo de água e energia, maiores perdas de nutrientes por escoamento superficial e percolação profunda, ao mesmo tempo em que podem apresentar plantas com déficits hídricos, em proporção significativa da área irrigada, (SCALOPPI \& DIAS, 1996).

O objetivo desse trabalho é avaliar a uniformidade de aplicação de água dos sistemas de irrigação por aspersão convencional, microaspersão e gotejamento em lotes do Perímetro Irrigado Senador Nilo Coelho, visando contribuir para otimização da produtividade, paralelo a sustentabilidade dos recursos hídricos.

\section{Material e métodos}

A pesquisa foi conduzida no período de julho a dezembro de 2007 no Perímetro Irrigado Nilo Coelho em Petrolina - PE. A região situa-se no submédio São Francisco, entre as coordenadas geográficas $40^{\circ} 50^{\prime} \mathrm{e}$ $40^{\circ} 23^{\prime \prime}$ de Longitude Oeste e $09^{\circ} 14^{\prime}$ e $09^{\circ} 27^{\prime}$ de Latitude Sul e se estende desde o município de Casa Nova (norte do Estado da Bahia) até o município de Petrolina (Sudoeste do Estado de Pernambuco), onde se encontra $80 \%$ da área do perímetro. Segundo a Companhia de Desenvolvimento dos Vales do São Francisco e Parnaíba (CODEVASF) compreende uma área de 18.858 ha irrigável, sendo ocupada 12.520 ha por lotes familiares e 6.043 ha por lotes empresariais.

Do total de lotes do Perímetro Irrigado Nilo Coelho foram escolhidos ao acaso e avaliados dezoito, sendo que dez com o sistema de microaspersão, três com gotejamento e cinco com aspersão convencional, para fins de cálculo dos coeficientes de uniformidade de distribuição de água.

\section{Descrição da área}

O clima da região que compreende o pólo Petrolina-PE/Juazeiro-BA é do tipo BSwh' isto é, seco, muito quente e com estação chuvosa no verão, atrasando-se para o outono, segundo a classificação de Köpen. Um dos maiores problemas das regiões semiáridas é a irregularidade das chuvas, aliada à ocorrência de temperaturas elevadas, ocasionando grandes taxas de evapotranspiração, levando a deficiência hídrica extrema.

Na região do Vale do Submédiodo São Francisco, tem sido observado ocorrência de temperaturas nos períodos diurnos e noturnos de $30^{\circ} \mathrm{C}$ e $25^{\circ} \mathrm{C}$, respectivamente. Com relação à temperatura do ar, segundo Teixeira (2010) a mínima média varia de 18,4 a $22,2{ }^{\circ} \mathrm{C}$, e a máxima de 29,6 a $33,9{ }^{\circ} \mathrm{C}$. Constata-se uma pequena variabilidade interanual, devida à proximidade da região em relação ao equador terrestre, sendo julho o mês mais frio e novembro o mês mais quente do ano.

A precipitação pluvial é o elemento meteorológico de maior variabilidade espacial e temporal. Nos últimos 30 anos, em Petrolina$\mathrm{PE}$, o total anual médio é da ordem de $567 \mathrm{~mm}$. O período chuvoso concentra-se entre os meses de novembro e abril, com $90 \%$ do total anual. A quadra chuvosa, de janeiro a abril, contribui com $68 \%$ do total anual, destacando-se o mês de março e o de agosto como o mais e o menos chuvoso, respectivamente, com totais médios de 136,2 mm. (TEIXEIRA \& LIMA FILHO, 2004)

\section{Solos}

No Vale do São Francisco, no Pólo Petrolina-PE/Juazeiro-BA, são mais frequientes as classes dos Vertissolos, Argissolos, Latossolos e Neossolos Quartzarênicos. (SILVA; GOMES, 2004) 
Wagner Willen Cavalcanti Araquam \& Luís Fernando de Souza Magno Campeche

\section{Água}

A principal fonte hídrica desse perímetro é o rio São Francisco. Teve funcionamento no início de de 1984 e início da cogestão com o Distrito de Irrigação de Bebedouro em 1989.

A captação dos recursos hídricos deriva da Barragem de Sobradinho, de propriedade da Companhia Hidroelétrica do São Francisco (CHESF). Os trabalhos de operação e manutenção dos Projetos Irrigados são de responsabilidade de Distritos de Irrigação localizados nos Perímetros, sendo associações civis sem fins lucrativos que congregam os usuários na área de abrangência dos Projetos.

O Perímetro Irrigado Nilo Coelho de acordo com a CODEVASF possui $158 \mathrm{~km}$ de canais; $818 \mathrm{~km}$ de adutoras; $711 \mathrm{~km}$ de estradas; $262 \mathrm{~km}$ de drenos; 39 estações de bombeamento.

\section{Avaliação dos sistemas de irrigação}

A metodologia utilizada para avaliação da irrigação foi determinada de acordo com o tipo de sistema:

\section{Metodologia de avaliação para irrigação localizada}

Para avaliação do sistema foi realizado teste de campo para verificar a uniformidade de aplicação de água pelo sistema de irrigação nos lotes. Utilizando recipientes e cronômetros; foram coletados volumes de água aplicada ao longo das linhas laterais para determinado tempo de funcionamento. Foram obtidos dados de vazão em três pontos ao longo da linha, sendo: no início, na posição mediana e final da linha. Para cada medição de vazão foram realizadas três repetições.

A metodologia descrita permitiu a obtenção dos indicadores de uniformidade de aplicação de água, Coeficiente de Uniformidade de Christiansen - CUC, Coeficiente de Uniformidade de Distribuição CUD e Coeficiente de Uniformidade Estatístico - CUE.

O CUC (Equação 1), é a mais popular medida de uniformidade, amplamente utilizada em irrigação por aspersão e localizada.

$$
C U C(\%)=100 *\left(1-\frac{\sum|X i-\bar{X}|}{N * \bar{X}}\right)
$$

Em que:

$\mathrm{Xi}=$ precipitação observada em cada pluviômetro, $\mathrm{mm}$;

$\bar{X}$ = média das precipitações, $\mathrm{mm}$; $\mathrm{N}$ = Número de pluviômetros;
(Eq. 1)

O Coeficiente de Uniformidade de Distribuição de água (CUD) - com base no menor quartil é representado na Equação 2, muito utilizado na irrigação localizada e enfatiza a importância de se suprir à cultura pelo menos a lâmina média de irrigação.

$$
C U D(\%)=\frac{\bar{x}}{\bar{X}} * 100
$$

Em que:

$\bar{x}=$ Média de $25 \%$ do total de pluviômetros, com as menores precipitações, $\mathrm{mm}$; $\mathrm{C}=$ Média de todos os pluviômetros.
O Coeficiente de Uniformidade Estatístico (CUE), é descrito pela Equação 3 e baseia-se no coeficiente de variação da lâmina de água aplicada ou vazão dos emissores, substituída por Bralts et al. (1987). 
Wagner Willen Cavalcanti Araquam \& Luís Fernando de Souza Magno Campeche

$$
C U E(\%)=100 *\left(1-\frac{S}{\bar{X}}\right)
$$

Em que:

$\bar{X}=$ Média de todos os pluviômetros, $\mathrm{mm}$;

$\mathrm{S}=$ Desvio-padrão dos dados de precipitação;

$$
S=\sqrt{\left(\frac{\sum_{i=1}^{N}|X i-\bar{X}|^{2}}{N}\right)}
$$

Em que:

$\mathrm{Xi}=$ precipitação observada em cada pluviômetro, mm;

Metodologia de avaliação da irrigação por aspersão

Para avaliação do sistema de irrigação fez-se uso da seguinte metodologia: Instalou-se um conjunto de 24 pluviômetros, eqüidistantes, no espaçamento de $3,00 \times 3,00 \mathrm{~m}$, formando um quadro com quatro linhas e seis colunas entre quatro aspersores, sendo dois de uma linha de distribuição e dois de outra linha. A área entre os 4 aspersores foi dividida em subáreas quadradas, de iguais dimensões. Os coletores de precipitação são colocados no centro de cada subárea. Assim, o volume ou lâmina coletada, em cada pluviômetro, representa a precipitação em cada subárea.

$\mathrm{O}$ resultado deste teste representa a interação entre os aspersores, pois está sendo avaliando, o efeito da sobreposição dos aspersores sobre uma mesma área. Colocou-se para funcionar as duas linhas de distribuição por um período de uma hora. Estes conjuntos
A variável (S) é o desvio-padrão dos dados de precipitação, sendo determinado pela seguinte equação:

$\bar{X}=$ média das precipitações, $\mathrm{mm}$;

$\mathrm{N}$ = Número de pluviômetros;

de pluviômetros foram distribuídos em 3 posições ao logo da linha de distribuição, ficando assim distribuídos entre os aspersores 2 e 3; 6 e 7; 9 e 10. Para os aspersores selecionados foram coletados dados de vazão utilizando-se um tubo coletor que conduziu toda à água aspergida para um balde de 13 litros e simultaneamente foi cronometrado o tempo para completar este volume, para cada medição foi realizada três repetições.

O procedimento descrito acima foi utilizado com objetivo de coletar os dados de vazão dos emissores para cálculo e análise dos coeficientes de uniformidade de Christiansen CUC, Coeficiente de uniformidade de Distribuição com base no menor quartil - CUD e o Coeficiente de Uniformidade Estatístico CUE .

Na Tabela 2 estão os dados de referência para fins de classificação dos coeficientes CUC e CUD estudados.

Tabela 2. Classificação dos valores do Coeficiente de Uniformidade de Christiansen(CUC) e do Coeficiente

\begin{tabular}{|c|c|c|}
\hline 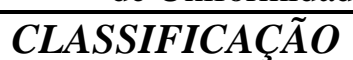 & $C U C(\%)$ & CUD (\%) \\
\hline Excelente & $>90$ & $>84$ \\
\hline Bom & $80-90$ & $68-84$ \\
\hline Razoável & $70-80$ & $52-68$ \\
\hline Ruim & $60-70$ & $36-52$ \\
\hline
\end{tabular}
de Uniformidade de Distribuição (CUD). 
Wagner Willen Cavalcanti Araquam \& Luís Fernando de Souza Magno Campeche

\begin{tabular}{lll}
\hline Inaceitável & $<60$ & $<36$ \\
\hline
\end{tabular}

Fonte: Mantovani (2001)

$\mathrm{Na}$ Tabela 3 está representado o aceitabilidade do CUD e CUE observado, de parâmetro de classificação quanto a acordo com a ASAE (1996).

Tabela 3. Grau de aceitabilidade CUE/CUD.

\begin{tabular}{lll}
\hline GRAU DE ACEITABILIDADE & $\boldsymbol{C U E}$ & $\boldsymbol{C U D}$ \\
\hline Excelente & $100-95$ & $100-94$ \\
Bom & $90-85$ & $87-81$ \\
Normal & $80-75$ & $75-68$ \\
Ruim & $70-65$ & $62-56$ \\
Inaceitável & $<60$ & $<50$ \\
\hline
\end{tabular}

Fonte: ASAE (1996)

\section{Resultados e Discussão}

Os dados referentes ao Coeficiente de Uniformidade de Christiansen (CUC), ao Coeficiente de Uniformidade de Distribuição (CUD) e ao Coeficiente de Uniformidade Estatístico (CUE), estão apresentados nas tabelas e gráficos a seguir, de acordo com o

\section{Irrigação localizada - microaspersão}

$\mathrm{Na}$ Tabela 4 estão apresentados os resultados dos coeficientes: CUC, CUD e CUE; para o sistema de microaspersão por lote avaliado. sistema e lote aferido.

Tabela 4 - Coeficiente de uniformidade de distribuição de água (CUC, CUD e CUE) para o sistema de microaspersão.

\begin{tabular}{|c|c|c|c|c|c|c|c|c|c|c|}
\hline \multicolumn{11}{|c|}{ IRRIGAÇÃO POR MICROASPERSÃO } \\
\hline & $\begin{array}{l}\text { LOTE } \\
609\end{array}$ & $\begin{array}{l}\text { LOTE } \\
309\end{array}$ & $\begin{array}{l}\text { LOTE } \\
392\end{array}$ & $\begin{array}{l}\text { LOTE } \\
521\end{array}$ & $\begin{array}{l}\text { LOTE } \\
765\end{array}$ & $\begin{array}{l}\text { LOTE } \\
1000\end{array}$ & $\begin{array}{l}\text { LOTE } \\
1079\end{array}$ & $\begin{array}{l}\text { LOTE } \\
1141\end{array}$ & $\begin{array}{l}\text { LOTE } \\
540\end{array}$ & $\begin{array}{l}\text { LOTE } \\
1653\end{array}$ \\
\hline $\begin{array}{l}\text { CUD } \\
(\%)\end{array}$ & 64,27 & 72,76 & 91,95 & 89,33 & 71,12 & 94,27 & 95,47 & 92,14 & 78,77 & 93,98 \\
\hline $\begin{array}{l}\text { CUC } \\
(\%)\end{array}$ & 78,43 & 84,74 & 92,89 & 92,75 & 82,69 & 96,28 & 96,41 & 93,89 & 86,08 & 96,22 \\
\hline $\begin{array}{l}\text { CUE } \\
(\%)\end{array}$ & 73,75 & 77,98 & 92,28 & 90,00 & 80,16 & 95,63 & 95,98 & 91,43 & 82,23 & 95,17 \\
\hline
\end{tabular}

Dentre os valores dos coeficientes avaliados o CUD apresentou o menor valor obtido em campo, entretanto em sistemas de irrigação localizada, segundo López et al (1992), ele é o mais utilizado na avaliação, pois este possibilita um tratamento mais rigoroso a problemas de distribuição de água, que ocorrem ao longo da linha lateral.
Resultados semelhantes foram obtidos por Martins (2009), Peixoto et. al (2005) e Cordeiro (2006) em sistemas de irrigação por microaspersão.

Na Tabela 5 pode ser observado a classificação dos lotes avaliados com microaspersão, de acordo com o critério qualitativo proposto por Mantovani (2001) e ASAE (1996): 
Wagner Willen Cavalcanti Araquam \& Luís Fernando de Souza Magno Campeche

Tabela 5 - Apresentação qualitativa dos resultados obtidos para os coeficientes de uniformidade de distribuição de água em cada lote para o sistema de microaspersão.

\begin{tabular}{llll}
\hline $\begin{array}{l}\text { SISTEMA } \\
\text { LOCALIZADO }\end{array}$ & LOTE & $\begin{array}{l}\text { CLASSIFICAÇÃO } \\
\text { CUC-CUD }\end{array}$ & $\begin{array}{l}\text { GRAU DE } \\
\text { ACEITABILIDADE } \\
\text { CUE-CUD }\end{array}$ \\
\hline & & Razoável & Ruim-Normal \\
& $\mathbf{6 0 9}$ & Bom & Normal \\
& $\mathbf{3 0 9}$ & Excelente & Bom-Excelente \\
Microaspersão & $\mathbf{5 2 1}$ & Excelente & Bom-Excelente \\
& $\mathbf{7 6 5}$ & Bom & Normal \\
& $\mathbf{1 0 0 0}$ & Excelente & Excelente \\
& $\mathbf{1 0 7 9}$ & Excelente & Excelente \\
& $\mathbf{1 1 4 1}$ & Excelente & Bom-Excelente \\
& $\mathbf{5 4 0}$ & Bom & Normal-Bom \\
& $\mathbf{1 6 5 3}$ & Excelente & Excelente \\
\hline
\end{tabular}

De acordo com Merriam e Keller (1978), o CUD que variar entre 80 e $90 \%$ é considerado bom, para um sistema que já se encontra em operação por algum tempo. „, que é o caso de vários lotes avaliados.

Os coeficientes de uniformidade estatísticos encontrados, excluindo os lotes 309 e 609 abaixo de $80 \%$, de maneira geral, foram classificados de Bom a Excelente, inclusive também para gotejo, muito semelhantes ao dados observados por Carvalho (2009) e Carvalho (2010).

\section{Irrigação localizada - gotejamento}

Na Tabela 6 pode ser observadoos resultados dos coeficientes: CUC, CUD e CUE; para o sistema de gotejamento, por lote aferido.

Tabela 6 - Coeficiente de uniformidade de distribuição de água (CUC, CUD e CUE) para o sistema de gotejamento.

\begin{tabular}{lccc}
\hline \multicolumn{5}{c}{ IRRIGAÇÃO POR GOTEJAMENTO } \\
\hline & LOTE $\mathbf{1 1 4 7}$ & LOTE 541 & LOTE 648 \\
CUD (\%) & 82,38 & 88,33 & 74,23 \\
CUC (\%) & 88,46 & 93,52 & 84,39 \\
CUE (\%) & 85,97 & 91,11 & 82,39 \\
\hline
\end{tabular}

Os valores de CUC encontrados são inferiores ainda aos resultados obtidos por Oliveira et al. (2003), que tiveram uma média de CUC de 96,6\%, em sistema de irrigação por gotejamento. $\mathrm{O}$ uso do CUC permite a obtenção de resultados bastante confiáveis, porém requer a medição da vazão de todos os emissores de uma unidade operacional, o que despende de muito tempo e mão-de-obra.

Segundo Mantovani \& Ramos (1994)apud Carvalho (2010), quanto maior o valor do CUC, menor é a lâmina de irrigação necessária para alcançar a produção máxima e de acordo com Bernardo (1995), o limite mínimo de CUC aceitável em um sistema de irrigação por gotejamento é de $80 \%$.

$\mathrm{Na}$ Tabela 7 está representada a classificação dos lotes com sistema de gotejamento, conforme o critério qualitativo apresentado por Mantovani (2001) e ASAE (1996): 
Wagner Willen Cavalcanti Araquam \& Luís Fernando de Souza Magno Campeche

Tabela 7 - Apresentação qualitativa dos resultados obtidos para os coeficientes de uniformidade de distribuição de água em cada lote para o sistema de gotejamento.

\begin{tabular}{llll}
\hline $\begin{array}{l}\text { SISTEMA } \\
\text { LOCALIZADO }\end{array}$ & LOTE & $\begin{array}{l}\text { CLASSIFICAÇÃO } \\
\text { CUC-CUD }\end{array}$ & $\begin{array}{l}\text { GRAU DE } \\
\text { ACEITABILIDADE } \\
\text { CUE-CUD }\end{array}$ \\
\hline \multirow{2}{*}{ Gotejo } & 1147 & Bom & Bom \\
& 541 & Excelente & Bom-Excelente \\
& 648 & Bom & Normal-Bom \\
\hline
\end{tabular}

Portanto, de acordo com os valores dos coeficientes de uniformidade de distribuição para irrigação localizada, apresentado nas tabelas 4 e 6 , verificou-se que quase todos os lotes avaliados, aproximadamente 92\%, apresentaram resultados satisfatórios, segundo Mantovani (2001) e normas da ASAE (1996). Desse percentual, pouco mais de $38 \%$ foi classificado em Bom e Normal e quase $54 \%$ foi registrado entre Bom e Excelente para o grau de aceitabilidade (Figura 1), que são superiores aos valores convencionalmente e recomendados, CUD > 84\%, para o funcionamento adequado de um sistema de irrigação localizada, (MANTOVANI, 2001).

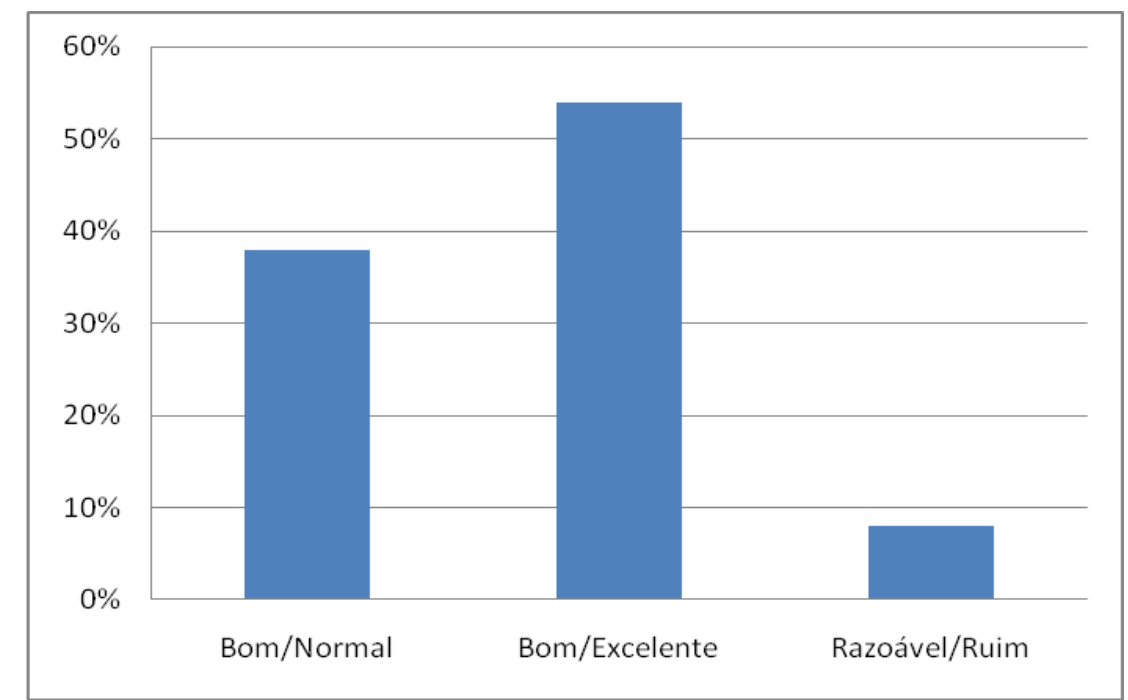

Figura 1 - Classificação CUC-CUD/CUE-CUD para irrigação localizada.

O lote 609 foi o único que não atingiu valor aceitável do grau exigido para o sistema, ficando entre razoável e ruim para uniformidade de distribuição.

Conforme Zocoler (2005), valores de CUC inferiores a $90 \%$ em sistemas localizados, só podem ser admitidos se a precipitação pluvial for significativa durante o cultivo, ou se a diminuição dos custos do sistema, com a redução da uniformidade, compensar a diminuição da receita devido à redução na produção da cultura, que não é o caso da região estudada.

\section{Irrigação por aspersão}

Para irrigação por aspersão, cinco lotes foram avaliados cujos resultados estão apresentados na Tabela 7: 
Wagner Willen Cavalcanti Araquam \& Luís Fernando de Souza Magno Campeche

Tabela 7 - Coeficiente de uniformidade de distribuição de água (CUC, CUD e CUE) para o sistema de aspersão convencional.

\begin{tabular}{lccccc}
\hline \multicolumn{7}{c}{ IRRIGAÇÃO POR ASPERSÃO } \\
\hline \multirow{2}{*}{ CUD (\%) } & LOTE 1029 & LOTE 1059 & LOTE 1482 & LOTE 1515 & LOTE 1164 \\
CUC (\%) & 47,03 & 40,30 & 63,31 & 68,00 & 42,75 \\
CUE (\%) & 60,91 & 55,80 & 73,21 & 77,51 & 54,68 \\
& 46,03 & 41,86 & 62,61 & 72,22 & 35,04 \\
\hline
\end{tabular}

Os valores de CUC, CUD e CUE, variaram entre 35,04 e $77,51 \%$, que para casos específicos como os lotes 1482 e 1515 , segundo Merrian \& Keller (1978) seriam aceitáveis para irrigação complementar em frutíferas com raízes profundas. Porém, não seriam recomendáveis para a irrigação por aspersão de culturas de alto valor econômico, com sistema radicular pouco profundo, nos quais necessitam que os valores de CUC e CUD sejam superiores a 88,0 e $80,0 \%$, respectivamente.

Segundo Gomes (1994), admitem-se valores de CUC $<80 \%$, se a área a irrigar recebe chuvas durante o período de irrigação, que não é o caso do Vale do São Francisco com suas chuvas irregulares; se a planta possui sistema radicular profundo, ou se a economia obtida no custo de implantação do projeto, compensar a redução do rendimento da cultura irrigada.

Relacionando-se CUC e CUD, Resende (1992) afirma que o fato de CUD ser sempre menor que CUC é inerente às variáveis das equações utilizadas na determinação desses coeficientes, pois no cálculo de CUD consideram-se apenas $25 \%$ da área que recebeu menos água.

Na Tabela 9 pode ser observadaa classificação dos lotes avaliados com sistema de aspersão convencional, segundo o critério qualitativo proposto por Mantovani (2001) e ASAE(1996):

Tabela 09 - Apresentação qualitativa dos resultados obtidos para os coeficientes de uniformidade de distribuição de água em cada lote para irrigação por aspersão convencional.

\begin{tabular}{llll}
\hline SISTEMA & LOTE & $\begin{array}{l}\text { CLASSIFICAÇÃO } \\
\text { CUC-CUD }\end{array}$ & $\begin{array}{l}\text { GRAU DE } \\
\text { ACEITABILIDADE } \\
\text { CUD-CUE }\end{array}$ \\
\hline & & Ruim & Inaceitável \\
ASPERSÃO & 1029 & Inaceitável-Ruim & Inaceitável \\
CONVENCIONAL & 1489 & Razoável & Inaceitável-Ruim \\
& 1515 & Razoável & Ruim-Normal \\
& 1164 & Inaceitável-Ruim & Inaceitável \\
\hline
\end{tabular}

De forma geral, a uniformidade de distribuição medida através dos coeficientes, CUC, CUD e CUE, foram inferiores aos encontrados por Paulino et al. (2009), apresentando um semelhante aspecto de sensibilidade às variações na distribuição de água no CUD, como também observados por Souza et. al. (2006).

Na irrigação por aspersão convencional, os lotes avaliados representaram $28 \%$ do total de lotes aferidos. Diferentemente da irrigação localizada, $80 \%$ desse sistema apresentou péssimos resultados (Figura 2) com o grau de 
Wagner Willen Cavalcanti Araquam \& Luís Fernando de Souza Magno Campeche

aceitabilidade entre ruim e inaceitável necessitando assim, de manutenção e/ou redimensionamento. Já o lote 1515 , no qual foi classificado entre razoável e normal (Tabela 9), que se trata de um resultado aceitável na literatura, porém não satisfatório para as condições de baixa pluviosidade da região e da fruticultura de alto custo de produção.

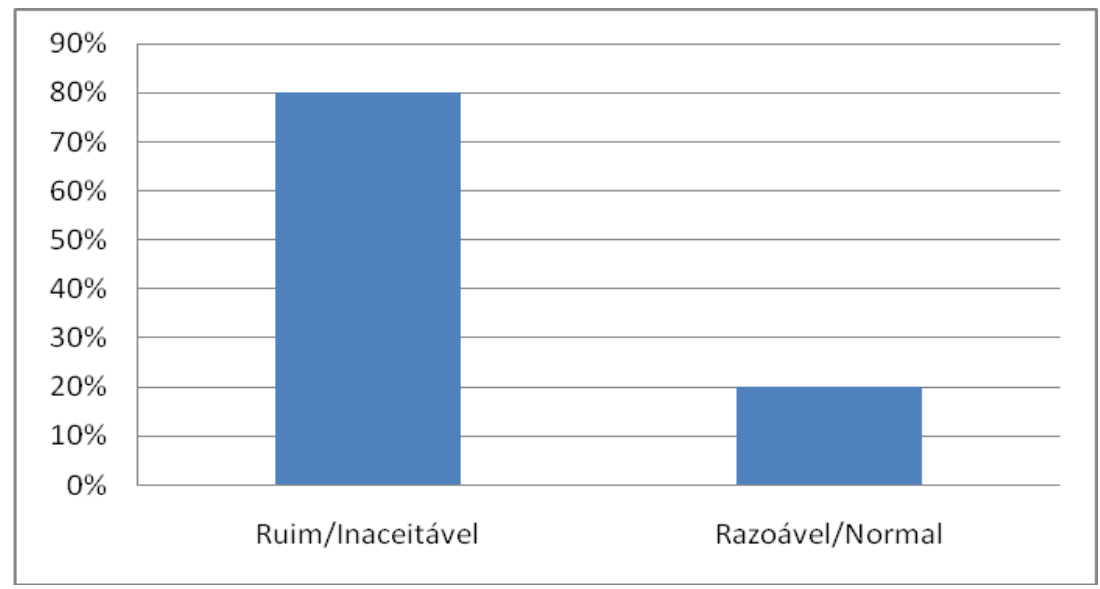

Figura 2 - Classificação CUC-CUD/CUE-CUD para irrigação por aspersão convencional.

Os resultados obtidos, a partir do uso dos três coeficientes CUC, CUD e CUE é é confiável segundo Favetta et al. (2001) e Oliveira et al. (1993), que observaram uma alta correlação entre os mesmos. Dessa forma, verifica-se que os sistemas de irrigação, devido a sua desuniformidade de aplicação de água, podem está prejudicando as culturas e desencadeando uma série de problemas e despesas para lavoura.

A região do Vale do Submédio do São Francisco apresenta uma fruticultura de alto custo de produção. Logo, valores do CUC inferiores a $80 \%$ para irrigação por aspersão convencional e $90 \%$ para irrigação localizada, poderá implicar em um desequilíbrio produtivo considerável da cultura e consequentemente redução na produtividade, mediante o mercado competitivo.

\section{Conclusões}

Os sistemas de irrigação localizada apresentaram maior uniformidade de distribuição de água, do que nos sistemas por aspersão convencional.
A maioria dos lotes com gotejamento e microaspersão está trabalhando dentro de uma margem aceitável de funcionamento.

A maior parte dos sistemas de aspersão convencional aferidos apresentou baixos coeficientes de uniformidade.

\section{Referências}

\section{ASAE. Field Evaluation of Microirrigation} Systems. St. Joseph, p.792-797. 1996.

BERNARDO, S. Manual de irrigação. 6. ed. rev. e ampl. Viçosa: UFV, Imprensa Universitária, 1995. 657 p.

BOMAN, B. J. Distribution patterns of microirrigation spinner and spray emitters. Applied Engineering in Agriculture. St. Joseph v.5, n.2, p.50-56. 1989.

BRALTS, V. F.; EDWARD, D.M.; WU, I.P. Drip irrigation design and evaluation based on statistical uniformity concept. In: HILLEL, D. 
Wagner Willen Cavalcanti Araquam \& Luís Fernando de Souza Magno Campeche

(Ed). Advances in irrigation. Orlando: Academic Press, 1987. v.4, p.67-117.

BRASIL. Ministério da Integração Nacional. CODEVASF. Senador Nilo Coelho. Disponível em: $<$ http://www.codevasf.gov.br/principal/perimet ros-irrigados/elenco-de-projetos/senador-nilocoelho> Acesso em 14 dez. 2010.

CABELLO, F. P. Riegos localizados de alta frecuencia goteo, microaspesion e exudacion. Madrid: Ed. Mundi- Prensa, 1986. 461p.

CARVALHO, C. M. de; ELOI, W. M.; LIMA, S. C. R. V.; PEREIRA, J. M. G. Desempenho de um sistema de irrigação por gotejamento na cultura da goiaba. Revista Irriga. Botucatu, v. 11, n. 1, p. $36-46,2006$.

CARVALHO, J. F. Manejo de irrigação no cultivo do repolho (Brassica oleracea var. capitata L.) em solo aluvial no Semi-árido. 118f. Dissertação (Mestrado em Engenharia Agrícola) - Universidade Federal Rural de Pernambuco, Recife, 2009.

CARVALHO, J. F. et al. Avaliação da uniformidade de sistema de irrigação localizada tipo gotejamento em cultivo de feijoeiro. In: X JORNADA DE ENSINO, PESQUISA E EXTENSÃO - JEPEX 2010, Recife. Recife: UFRPE, 2010.

CHRISTIANSEN, J.E. Irrigation by sprinkling. Berkeley: University of California, 1942. 124p.

CHRISTOFIDIS, D. Água na produção de alimentos: o papel da academia e da indústria no alcance do desenvolvimento sustentável. Revista de Ciências exatas. Taubaté, v. 12, n. 1, p. 37-46, 2006.

COELHO, F. E.; COELHO FILHO, M. A.; OLIVEIRA, S. L. de. Agricultura irrigada: eficiência de irrigação e de uso de água.
Revista Bahia Agrícola. Cruz das almas, v.7, n.1, set. 2005.

CORDEIRO, E. A. Diagnóstico e manejo da irrigação na cultura do mamoeiro na região norte do Estado do Espírito Santo. 2006. 100 f. Tese (Doutorado em Engenharia Agrícola) Universidade Federal de Viçosa, Minas Gerais, 2006.

CRIDDLE, W.D.; DAVIS, S.; PAIR, C.H.; SHOCKLEY, D.G. Methods for evaluating irrigation systems. Washington, DC: Soil Conservation Service - USDA, 1956. 24p. (Agricultural Handbook, 82).

Distrito de Irrigação Nilo Coelho. O perímetro Nilo Coelho. [Petrolina, 2010]. Disponível em: $<$ http://www.dinc.org.br/index.php?sessao=peri

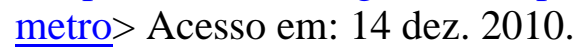

FAVETTA, G. M.; BOTREL, T. A. Uniformidade de sistemas de irrigação localizada: validação de equações. Scientia Agricola. Piracicaba, v. 58, n.2, p.427-430, abr./jun. 2001.

FRIZZONE, J. A. Irrigação por aspersão: uniformidade e eficiência. Piracicaba: ESALQ, 1992. 53 p. (Séria Didática).

GOMES, H. P. Engenharia de irrigação: Hidráulica dos sistemas pressurizados, aspersão e gotejamento. João Pessoa: Universidade Federal da Paraíba, 1994. 344p.

HEERMANN, D.F.; DUKE, H.R.; SERAFIM, A.M.; DAWSON, L.L. Distribution functions to represent centerpivot water distribution. Transactions of the ASAE, St. Joseph, v.35, n.5, p.1465-1472, 1992.

KELLER, J.; BLIESNER, R.D. Sprinkle and trickle irrigation. New York: van Nostrand Reinhold, 1990. 652p.

KELLER, J.; KARMELI, D. Trickle irrigation design parameters. Transaction of 
Wagner Willen Cavalcanti Araquam \& Luís Fernando de Souza Magno Campeche

the ASAE. St. Joseph, v.17, n.4, p.678-684, July/Aug., 1974.

LÓPEZ, J. R., ABREU, J. M. H.; REGALADO, A. P.; HERNÁNDEZ, J. F. G. Riego Localizado. Madrid, Espana: Mundi Prensa, 1992. 405p.

MANTOVANI, E. C. AVALIA: Programa de Avaliação da Irrigação por Aspersão e Localizada. Viçosa, MG: UFV, 2001.

MANTOVANI, E.C.; VILLALOBOS, F.J.; ORGAZ, F.; FERERES, E. Modelling the effects of sprinkler irrigation uniformity on crop yield. Agricultural Water Management, Amsterdam, v.27, n.3/4, p.243-257, 1995.

MARTINS, C. A. da S. Avaliação do desempenho de sistemas de irrigação em áreas cultivadas no Sul do Estado do Espírito Santo. 2009. 107 f. Dissertação (Mestrado em Produção Vegetal). Centro de Ciências Agrárias, UFES, Espírito Santo, 2009.

MATOS, J. A.; DANTAS NETO, J.; AZEVEDO, H.M.; AZEVEDO, C.A.V. Características hidráulicas do microaspersor Dan Sprinkler 2001. Irriga. Botucatu, v.1, n.3, p.30-44. 1996.

MERRIAN, J.L.; KELLER, J. Farm irrigation system evaluation: A guide for management. Logan: Agricultural and Irrigation Engineering Departament, Utah State University, 1978. 271p.

OLIVEIRA, et al. Uniformidade de distribuição de potássio num sistema de irrigação por gotejamento quando aplicado por diferentes injetores. Irriga. Botucatu, v. 8, n. 3, p. 234241, set-dez. 2003.

OLIVEIRA, L. F. C. de. et al. Avaliação da distribuição de vazão e hidráulica das linhas laterais de um sistema de irrigação por microaspersão. Engenharia Rural. Piracicaba, 4(único), p.37-58, jul/dez. 1993.
PAUlinO, M. A. de O et al. Avaliação da uniformidade e eficiência de aplicação de água em sistemas de irrigação por aspersão convencional. Revista Brasileira de Agricultura Irrigada. Fortaleza, v.3, n.2, p.48-54, 2009.

PEIXOTO, J. F. S.; CHAVES, L. H. G.; GUERRA, H. O. C. Uniformidade de distribuição da fertirrigação no distrito de irrigação Platô de Neópolis. Revista de Biologia e Ciências da Terra. Campina Grande, v. 5, n. 2, 2. Sem. 2005.

PERI,G.; HART, W.E.; NORUM, D.J. Optimal irrigation depths-a method of analysis. Journal of the Irrigation and Drainage Division. New York, v.105 n.4 343- 55, dec.1979.

RESENDE, R. Desempenho de um sistema de irrigação Pivô Central quanto à uniformidade e eficiência de aplicação de água, abaixo e acima da superfície do solo. 1992. 86 f. Dissertação (Mestrado em Agronomia) - Escola Superior de Agricultura "Luiz de Queiroz", Universidade de São Paulo, Piracicaba, 1992.

SANTOS, R. A. et al. Uniformidade de distribuição de água em irrigação por gotejamentos em sub-superfície instalado na cultura de pupunheiras (Bactris gasipaes H.B.K.). In: XXXII Congresso Brasileiro de Engenharia Agrícola - CONBEA, 2003, Goiânia: GO, jul/ago. 2003.

SCALOPPI, J. E.; DIAS, K. F. S. Relação entre a pressão de operação e a uniformidade de distribuição de água de aspersores rotativos por impacto. In: CONGRESSO BRASILEIRO DE ENGENHARIA AGRÍCOLA, 26, 1996, Bauru. Resumos... Bauru: Sociedade Brasileira de Engenharia Agrícola, 1996. CD Rom.

SILVA, C. A da.; SILVA, C. J. da. Avaliação de uniformidade em sistemas de irrigação 
Wagner Willen Cavalcanti Araquam \& Luís Fernando de Souza Magno Campeche

localizada. Revista Científica Eletrônica de Agronomia. Garça, n. 8, dez. 2005.

SILVA, M. S. L da; GOMES, T. C. de A. Cultivo da mangueira. Disponível em: http://sistemasdeproducao.cnptia.embrapa.br/F ontesHTML/Manga/CultivodaMangueira/mane jo.htm>. Acesso em 11 jan. 2011.

SOUZA, L. O. C.; MANTOVANI, E. C.; SOARES, A. A.; RAMOS, M. M.; FREITAS, P. S.L. Avaliação de sistemas de irrigação por gotejamento, utilizados na cafeicultura. Revista Brasileira de Engenharia Agrícola e Ambiental. Campina Grande, v.10, n.3, jul./set. 2006.

TARJUELO, J. M.; De JUAN, J. A.; VALIENTE, M.; GARCÍA, P. Model for optimal cropping patterns within the farm based on crop water production functions and irrigation uniformity. II: A case study of irrigation scheduling in Albacete, Spain. Agricultural Water Management, Amsterdam, v.31, n.1/2, p.145-163, 1996.

TEIXEIRA, A. H. de C. Informações agrometeorológicas do Polo Petrolina, PE/Juazeiro, BA - 1963 a 2009. Petrolina: Embrapa Semiárido, 2010. 21 p. (Embrapa Semiárido. Documentos, 233).

TEIXEIRA, A. H. de C.; LIMA FILHO, J. M. L. Cultivo da mangueira. Embrapa Semiárido: 2004. Disponível em: <http://sistemasdeproducao.cnptia.embrapa.br/ FontesHTML/Manga/CultivodaMangueira/clim a.htm>. Acesso em 11 jan. 2011.

TUNDISI, J. G. Água no século XXI: enfrentando a escassez. 2. ed. São Carlos: Rima, 2005. 248 p.

WALKER, W.R. Explicit sprinkler irrigation uniformity: efficiency model. Journal of the Irrigation and Drainage Division. ASCE, New York, v.105, n.2, p.129-36, june 1979.
WILCOX, J.C.; SWAILES, G.E. Uniformity of water distribution by some undertree orchard sprinklers. Scientific Agricultural, v.27, p.565-583, 1947.

ZOCOLLER, J. L. Avaliação de desempenho de sistemas de irrigação. Ilha Solteira - SP: UNESP, 2005. Disponível em <cwww.agr.feis.unesp.br/curso5.htm>. Acesso em 14 de out. de 2010.p 